\title{
Bone metabolism and the development of denosumab: a narrative review
}

\author{
Xiaohui Niu, Hairong Xu \\ Department of Orthopedic Oncology Surgery, Beijing Ji Shui Tan Hospital, Peking University, Beijing, China \\ Contributions: (I) Conception and design: X Niu; (II) Administrative support: X Niu; (III) Provision of study materials or patients: All authors; (IV) \\ Collection and assembly of data: All authors; (V) Data analysis and interpretation: All authors; (VI) Manuscript writing: All authors; (VII) Final \\ approval of manuscript: All authors. \\ Correspondence to: Xiaohui Niu. Department of Orthopedic Oncology Surgery, Beijing Ji Shui Tan Hospital, Peking University, No.31 Xin Jie Kou \\ East Street, Xi Cheng District, Beijing 100035, China. Email: niuxiaohui@263.net.
}

\begin{abstract}
The bone of human body provides many basic functions, including normal bone metabolism. Bone plays an important role in maintaining physiological homeostasis of the body. The balance between osteolysis and osteogenesis is important in bone metabolism. This process reflects the osteoblast and osteolytic cell function of coordination. RANKL/RANK/OPG pathway plays a central regulatory role in normal bone remodeling. RANKL/RANK/OPG regulates the axis of Bone, which is an important way to influence bone formation and dynamic equilibrium. In the process of tumor osteolytic bone metastasis and giant cell tumor (GCT) of bone, the expression of RANKL increases, which leads to excessive bone resorption. Denosumab can specifically block RANKL, thereby inhibiting the activity of osteoclasts and blocking the development of disease. Skeletal-related event (SRE) caused by bone metastases not only reduce patients' physical function and quality of life, but also increase the risk of death. How to optimize the management of bone metastasis and relieve the pain caused by bone metastasis has become a hot spot in the field of cancer. Based on a series of clinical studies, Denosumab has been approved by the U. S. Food and Drug Administration for osteoporosis, solid tumor bone metastasis, GCT of bone, malignant tumor hypercalcemia and other fields. In China, Denosumab was indicated to treat GCT of bone, as well as SRE caused by solid tumor bone metastases and multiple myeloma.
\end{abstract}

Keywords: Bone metabolism; RANKL/RANK/OPG pathway; Denosumab

Received: 11 December 2020; Accepted: 15 January 2021; Published: 31 January 2021.

doi: $10.21037 /$ tbcr-20-69

View this article at: http://dx.doi.org/10.21037/tbcr-20-69

\section{Bone and the balance of bone metabolism}

The human skeleton provides many basic functions, including mechanical support, vital organ protection, hematopoietic microenvironment, storage of calcium and other minerals (1). Bone is composed of many kinds of cells, collagen matrix and inorganic elements. Bone has hematopoietic cells and immune cells in the normal regulation of body (2). Bone is required to maintain physiological homeostasis in the body, including acidbase balance, calcium or phosphate retention, and other important mineral storage and release $(3,4)$.
Bone formation and renewal involves three important cells: osteoblasts, osteocytes, and osteoclasts $(5,6)$. Bone cells are derived from bone marrow stem cells, which are derived from mesenchymal stem cell cells. Bone matrix is produced during the differentiation process and plays an important role in human development, bone injury and normal bone remodeling throughout the life cycle. Most of the studies on osteoclast differentiation have been performed in vitro using cells from mouse bone marrow. It is assumed that properly stimulated bone marrow stromal cells expressing RANKL in vivo initiate osteoclast differentiation in bone marrow and those partially differentiated mononucleated osteoclast 
progenitors, like other hematopoietic cells, are released into circulation (7). Bone cells are osteoblasts embedded in the matrix, surrounded by collagen and inorganic salts. The cells related to osteolysis are mainly osteoclasts. Osteoclasts is derived from blood cells derived from macrophage precursors (hematopoietic precursors of the monocytemacrophage lineage) and plays a role in bone remodeling by absorbing bone matrix. Normal bone metabolism requires a balance between osteogenesis and osteolysis. In the body, normal bone precisely undergoes a four-stage cycle of bone resorption, bone repair, bone formation and dormancy. Both osteoblast and osteoclast cell types are affected by a variety of hormones, inflammatory mediators and growth factors $(8,9)$. An imbalance in the function of osteoblasts and osteoclasts can lead to bone abnormalities. An imbalance is characterized by an increase in bone mass (osteosclerosis) or a decrease (osteoporosis). We present the following article in accordance with the Narrative reporting checklist (available at http://dx.doi.org/10.21037/tbcr-20-69).

\section{The discovery of OPG}

In the 1990s, Amgen Inc. used cDNA to find anti-tumor drugs (10). In the course of the study, a protein was found to protect bone from osteolysis by osteoclasts, hence the name "Osteoprotegerin" (OPG) (11). In addition (12), OPG ligand (OPGL), which can bind to OPG, was screened by recombinant fusion technology, and a cytokine related to tumor necrosis factor (TNF), called RANKL (activator of $\mathrm{nf}-\mathrm{\kappa b}$ receptor activator ligand), was found. The receptor for RANKL was also identified in DC cells (DENDRITIC cell). RANK (a receptor activator of nuclear FACTORKAPPA B) was also found to be expressed in osteoclasts and their precursors. More coincidentally, the RANKL sequence is identical to the OPGL sequence. As a new secretory member of the Tumor necrosis factor receptor family, this new member lacks any obvious cell related signals, suggesting that it may play a role in the extracellular environment, transgenic mice expressing this secretory protein showed a general increase in bone mineral density (Ossification) and a decrease in osteoclasts. Recombinant OPG has a similar effect in normal mice, preventing osteoclast differentiation from precursor cells in a dose-dependent manner and preventing ovariectomyrelated bone loss in rats, and OPG prevents osteoclast differentiation, it acts as a humoral regulator of bone resorption (13-15).

RANK is expressed on the surface of dendritic cells and t cells and hematopoietic precursors and is the receptor for RANKL. The combination of RANK and RANKL may regulate dendritic cell function and $\mathrm{T}$ cell activation. RANK maps to human genome $18 \mathrm{q} 22.1$, and RANKL maps to human genome $13 \mathrm{q} 14$, the first TNFR and TNF ligand family members to map to these regions (16).

Osteoprotegerin, expressed in osteoblasts, can bind to and inhibit the function of RANKL, thus preventing bone loss and increasing osteogenesis. Many diseases are characterized by an imbalance of bone metabolism, both in local and in systemic situation. In the pathophysiological process, an increase in osteolysis is the result of the overproduction of RANK-activated osteoclast-like giant cells. Pathological overproduction of RANKL can be caused by a number of factors, such as direct tumor secretion. The stromal cells in giant cell tumor (GCT) of bone can produce excessive RANKL. Excess RANKL can also be caused by other factors that stimulate osteoblast secretion, such as endocrine, infection, bone disease, bone metastases, myeloma, and so on. However, taking measures to neutralize the excess RANKL does not kill the tumor, but inhibits absorption, upregulates OPG, increases osteogenesis, and reduces and repairs bone destruction.

A large number of OPG variants have been explored in preclinical models, among which the form of OPG monomer, Polyethylene glycol, and RANK-Fc fusion of RANK and antibody FC segments is only in the preclinical stage (15). But basic research has shown great commercial value for researchers, and the development of RANKL inhibitors is imperative. At the clinical stage, the modified version of the recombinant OPG combines the active region of OPG with the c-terminal of FC, which is the first type of OPG (FcOPG) in human trials. The glycosylated OPG-Fc (AMGN0007) combines the active region of OPG with the n-terminal of $\mathrm{FC}$, the half-life is about ten times longer and three to ten times more potent than the Fc-OPG (17-19). Given the potential safety risks of inducing an immune response to OPG in an individual population, Amgen stopped developing OPG-fc and turned to a method of producing RANKL.

\section{Development of human antibody to RANKL (denosumab)}

In a major advance in antibody therapy, the transgenic mice provided full humanized monoclonal antibody that could be produced quickly and with high affinity, the antibody subtypes of IgG2 have few deleterious molecular effects on cells expressing RANKL (such as ADCC and 


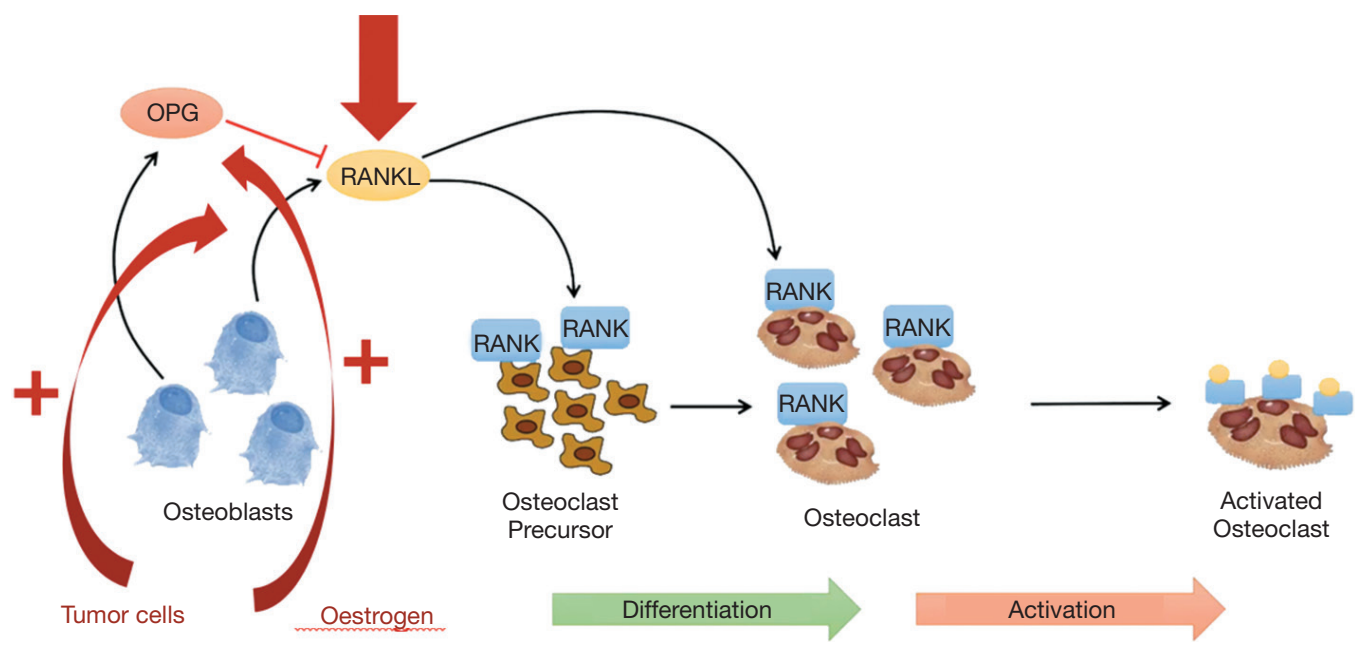

Figure 1 Osteoblasts secrete RANKL, which binds to RANKL on the surface of osteoclast precursors. In turn it differentiates into osteoclast. The surface of osteoclast still expresses RANKL. If RANKL continues to bind to RANK, it will differentiate into activated osteoclasts. This process is regulated by OPG, which binds to RANKL, blocking the subsequent activation of RANKL by osteoclasts. Denosumab mimics OPG's function. Tumor cells can stimulate the body to produce more RANKL, whereas oestrogen can stimulate the body to produce more OPG.

CDC, antibody-dependent cell-mediated Cytotoxicity, and complement-dependent cytotoxicity) (20). Natural IgG2 antibodies differ from IgG1 and IgG4 in their hinge disulfide bonds, are highly susceptible to Papain and lose their potency through hydrolysis, and Amgen has a well-developed patented technique for modifying the hinge region of IgG2 antibodies, the modified IgG2 antibody reduces isomer differences and increases resistance to Papain. On the basis of this technique, a new humanized monoclonal antibody was born, with a molecular weight similar to that of the recombinant OPG-Fc, which can be combined with soluble or membrane bound human RANKL, denosumab was more specific than human OPG-Fc and prolonged the half-life of denosumab significantly $(15,21,22)$.

During the developing process of RANKL inhibitor as a therapeutic candidate, the scientist at Immunex Company pursued a conceptual and design strategy similar to that used for the development of etanercept. However, following repeated dosing of human RANKFc in primates, activating autoantibody against RANK were detected that led to hypercalcaemia. This highlighted the potential risk of an immune response to endogenous RANK in patients, and RANKFc development was discontinued. An improved version of recombinant OPG was produced in bacteria as an amino-terminal IgG Fc region fused to the ligand binding domain, and was the first version of OPG that was tested in humans (Fc-OPG). A superior recombinant OPG-Fc fusion protein (a C-terminal immunoglobulin $\mathrm{Fc}$ fusion protein comprising residues 22-194) known as AMGN-0007 was produced in mammalian cell cultures and was found to be glycosylated. Upon testing in humans, its potency was determined to be at least 20 times better than the bacterial Fc-OPG protein. Denosumab (known at the time as AMG 162) is a human $\operatorname{IgG} 2 \kappa$, and demonstrated in vitro neutralizing activity; it also had a similar molecular mass to recombinant OPG-Fc fusion proteins but a modestly lower affinity for human RANKL (10). The mechanism of Denosumab could be illustrated by Figure 1 .

\section{Denosumab treatment for bone metastasis}

Based on a series of clinical studies, Denosumab has been approved by the U. S. Food and Drug Administration for osteoporosis, solid tumor bone metastasis, GCT of bone, malignant tumor hypercalcemia and other fields. In China, Denosumab is also approved for bone metastases. The incidence of bone metastases is as high as $65-75 \%$ in patients with advanced breast cancer (23). The efficacy of denosumab as a bone-modifying agent in the treatment of Bone metastases from advanced breast cancer has been demonstrated in clinical studies.

The 136 study was a multicenter phase III clinical trial involving 2,046 patients with bone metastases from advanced breast cancer and compared the efficacy of denosumab 
with zoledronic acid (24). The results showed that the time between denosumab and zoledronic acid to first SRE was significantly longer (less than 26.4 months), and the risk of multiple SRE was reduced by $23 \%$ during the study period. Denosumab group was also significantly better than zoledronic acid group in delaying the onset of moderate to severe pain by 3.9 months. The improvement in quality of life was also better in the denosumab group, with HRQoL improving $10 \%$ more patients than zoledronic acid. A Phase III study of Denosumab versus zoledronic acid in the treatment of advanced solid tumors and multiple myeloma included 1,597 patients, including 811 with bone metastases from lung cancer (25). In the Lung Cancer Subgroup, denosumab significantly prolonged survival by 1.2 months (8.9 versus 7.7 months) compared with zoledronic acid and reduced the risk of death by $20 \%$. Among the overall lung cancer patients, NSCLC, especially squamous cell carcinoma, had the most significant survival benefit and a $32 \%$ reduction in mortality risk. In addition, denosumab significantly delayed the median time of pain exacerbation (8.2 versus 4.8 months) compared with zoledronic acid in baseline pain-free or mild-pain solid tumor patients.

\section{Denosumab treatment for GCT of bone}

The exact pathogenesis of GCT of bone is unclear. The imaging findings of GCT of bone were osteolysis. It was suggested that the osteolysis process of GCT was dependent on the combination of RANKL (receptor activator of nuclear $\kappa b$-ligand) and RANK $(26,27)$. There are two kinds of cells in the pathological morphology of GCT of bone: monocytes and osteoclast-like multinucleated giant cells. There are two types of monocytes: spindle stromal cells and macrophage cells. Spindle stromal cells (fusiform stromal cells) are tumor cells of GCT of bone. Macrophage are precursors of osteoclast like cells that fuse together to form osteoclast like multinucleated giant cells. Osteoclastlike giant cells express RANK, while spindle stromal cells express RANKL and RANKL RANK, which activate RANK-RANKL pathway and induce osteolysis.

RANKL as a key factor in the activation of osteoclast functional pathway has aroused great interest of scientists. In the research of RANKL-RANK-OPG pathway, Amgen has done a lot of work. Amgen eventually developed a humanized anti RANKL monoclonal antibody. Denosumab can competitively bind RANKL secreted by stromal cells, thus significantly reducing or eliminating the recruitment of osteoclast-like giant cells, thus blocking the activity of osteoclast-like giant cells, avoiding the osteolysis process, and increasing the formation of new bone, to slow the progression of the tumor.

\section{Summary}

Bone-modifying agents are the main bone-targeting agents for the treatment of bone metastases, including bisphosphonates and Denosumab, which can control the development of bone metastases to a great extent. Denosumab is the first precisely targeted RANKL inhibitor that blocks the binding of RANK to RANKL. Due to the unique mechanism of action of Denosumab, the initial use may bring more benefits to patients.

\section{Acknowledgments}

Funding: None.

\section{Footnote}

Reporting Checklist: The authors have completed the Narrative reporting checklist. Available at http://dx.doi. org/10.21037/tbcr-20-69

Conflicts of Interest: Both authors have completed the ICMJE uniform disclosure form (available at http://dx.doi. org/10.21037/tbcr-20-69). The authors have no conflicts of interest to declare.

Ethical Statement: The authors are accountable for all aspects of the work in ensuring that questions related to the accuracy or integrity of any part of the work are appropriately investigated and resolved.

Open Access Statement: This is an Open Access article distributed in accordance with the Creative Commons Attribution-NonCommercial-NoDerivs 4.0 International License (CC BY-NC-ND 4.0), which permits the noncommercial replication and distribution of the article with the strict proviso that no changes or edits are made and the original work is properly cited (including links to both the formal publication through the relevant DOI and the license). See: https://creativecommons.org/licenses/by-nc-nd/4.0/.1.

\section{References}

1. Buckwalter JA, Cooper RR. Bone structure and function. 
Instr Course Lect 1987;36:27-48.

2. Sugiyama T, Nagasawa T. Bone Marrow Niches for Hematopoietic Stem Cells and Immune Cells. Inflamm Allergy Drug Targets 2012;11:201-6.

3. Kiberstis P. Bone Health in The Balance. Science 2000;289:1497.

4. Chen J, Qiu M, Dou C, et al. MicroRNAs in Bone Balance and Osteoporosis. Drug Dev Res 2015;76:235-45.

5. Lerner UH. Osteoblasts, Osteoclasts, and Osteocytes: Unveiling Their Intimate-Associated Responses to Applied Orthodontic Forces. Semin Orthod 2012;18:237-48.

6. Rubin JE, Rubin CT. The osteoblast, osteocyte, and osteoclast. Curr Opin Orthop 1997;8:34-42.

7. Kollet O, Dar A, Shivtiel S, et al. Osteoclasts degrade endosteal components and promote mobilization of hematopoietic progenitor cells. Nat Med 2006;12:657-64.

8. Suda I, Hirayama K. Degradation of methyl and ethyl mercury into inorganic mercury by hydroxyl radical produced from rat liver microsomes. Arch Toxicol 1992;66:398-402.

9. Mundy GR. Cytokines and growth factors in the regulation of bone remodeling. J Bone Miner Res 1993;8:S505-10.

10. Lacey DL, Boyle WJ, Simonet WS, et al. Bench to bedside: elucidation of the OPG-RANK-RANKL pathway and the development of denosumab. Nat Rev Drug Discov 2012;11:401-19.

11. Simonet WS, Lacey DL, Dunstan CR, et al. Osteoprotegerin: A Novel Secreted Protein Involved in the Regulation of Bone Density. Cell 1997;89:309-19.

12. Kong YY, Yoshida H, Sarosi I, et al. OPGL is a key regulator of osteoclastogenesis, lymphocyte development and lymph-node organogenesis. Nature 1999;397:315-23.

13. de Castro LF, Burke AB, Wang HD, et al. Activation of RANK/RANKL/OPG Pathway Is Involved in the Pathophysiology of Fibrous Dysplasia and Associated With Disease Burden. J Bone Miner Res 2019;34:290-4.

14. Lacey DL, Timms E, Tan HL, et al. Osteoprotegerin Ligand Is a Cytokine that Regulates Osteoclast Differentiation and Activation. Cell 1998;93:165-76.

15. Sordillo EM, Pearse RN. RANK-Fc: A therapeutic antagonist for RANK-L in myeloma. Cancer 2003;97:802-12.

16. Hsu Y-H, Niu T, Terwedow HA, et al. Variation in genes involved in the RANKL/RANK/OPG bone remodeling pathway are associated with bone mineral density at different skeletal sites in men. Hum Genet 2006;118:568-77.

17. Body JJ, Greipp P, Coleman RE, et al. A Phase I study of
AMGN-0007, a recombinant osteoprotegerin construct, in patients with multiple myeloma or breast carcinoma related bone metastases. Cancer 2003;97:887-92.

18. Bekker P. A Single SC dose of AMGN-0007, a recombinant construct of osteoprotegerin (OPG), inhibits bone resorption for a prolonged period in patients with multiple myeloma. Eur J Haematol 2003;70:270-1.

19. Bekker P. A Single SC dose of AMGN-0007, a recombinant construct of osteoprotegerin (OPG), inhibits bone resorption for a prolonged period in patients with multiple myeloma. Eur J Haematol 2003;70:270-1.

20. Raux S, Bouhamama A, Gaspar N, et al. Denosumab for treating aneurysmal bone cysts in children. Orthop Traumatol Surg Res 2019;105:1181-5.

21. Ottewell PD, Wang N, Brown HK, et al. OPG-Fc inhibits ovariectomy-induced growth of disseminated breast cancer cells in bone. Int J Cancer 2015;137:968-77.

22. Ominsky MS, Kostenuik PJ, Cranmer P, et al. The RANKL inhibitor OPG-Fc increases cortical and trabecular bone mass in young gonad-intact cynomolgus monkeys. Osteoporos Int 2007;18:1073-82.

23. Awan AA, Hutton B, Hilton J, et al. De-escalation of bone-modifying agents in patients with bone metastases from breast cancer: a systematic review and meta-analysis. Breast Cancer Res Treat 2019;176:507-17.

24. Stopeck AT, Lipton A, Body JJ, et al. Denosumab Compared With Zoledronic Acid for the Treatment of Bone Metastases in Patients With Advanced Breast Cancer: A Randomized, Double-Blind Study. J Clin Oncol 2010;28:5132-9.

25. Henry D, Vadhan-Raj S, Hirsh V, et al. Delaying skeletalrelated events in a randomized phase 3 study of denosumab versus zoledronic acid in patients with advanced cancer: an analysis of data from patients with solid tumors. Support Care Cancer 2014;22:679-87.

26. Chawla S, Blay JY, Rutkowski P, et al. Denosumab in patients with giant-cell tumour of bone: a multicentre, open-label, phase 2 study. Lancet Oncol 2019;20:1719-29.

27. Balke M, Hardes J. Denosumab: a breakthrough in treatment of giant-cell tumour of bone? Lancet Oncol 2010;11:218-9.

doi: $10.21037 /$ tbcr-20-69

Cite this article as: $\mathrm{Niu} \mathrm{X}, \mathrm{Xu} \mathrm{H}$. Bone metabolism and the development of denosumab: a narrative review. Transl Breast Cancer Res 2021;2:7. 